Journal of

Epilepsy and

Clinical

Neurophysiology

J Epilepsy Clin Neurophysiol 2006; 12(4):207-218

\title{
Percepção de Estigma na Epilepsia
}

\author{
Paula Teixeira Fernandes*, LiMin Li** \\ Departamento de Neurologia - Faculdade de Ciências Médicas (UNICAMP)
}

\begin{abstract}
RESUMO
Introdução: Pessoas com epilepsia frequentemente vivenciam o estigma, muitas vezes mais prejudicial que a própria condição em si. De maneira geral, pode-se dizer que a epilepsia é uma das condições que mais afeta o comportamento e a qualidade de vida, não só da pessoa que tem epilepsia, mas também da família toda, especialmente devido ao estigma existente. Por isso, dizemos que a epilepsia causa um impacto bio-psicosocial na vida das pessoas. Porém, este aspecto do estigma na epilepsia é pouco abordado, especialmente em países em desenvolvimento, como o Brasil, onde superstições, atitudes negativas e falta de informação dificultam a relação da comunidade com a epilepsia. Objetivo: Este artigo tem o objetivo de discutir aspectos relevantes do estigma na epilepsia: conceituação e modelos de estigma na área médica e social; estigma e qualidade de vida; fatores operantes; aspectos neurobiológicos e estratégias para se lidar com o estigma na epilepsia. Conclusões: Este artigo mostrou uma visão geral do estigma englobando seus diferentes aspectos. Pelo fato de ser um conceito multifatorial, o combate ao estigma requer também uma intervenção ampla, envolvendo as áreas médica, psicológica e social. $\mathrm{O}$ entendimento do processo do estigma contribui para uma mudança da interpretação social da epilepsia, rumo a construção de uma sociedade mais justa e tolerante, na qual as diferenças sejam respeitadas.
\end{abstract}

Unitermos: estigma, qualidade de vida, epilepsia.

\begin{abstract}
Perception epilepsy' stigma

Introduction: People with epilepsy often need to deal with the stigma, usually worse than the epilepsy itself. In general, epilepsy is a condition that affects the behavior and the quality of life, not only for the person with epilepsy, but also for the entire family, especially because of stigma. For this reason, we can say that epilepsy has an bio-psycho-social impact on the people's life. Paradoxically, this facet of epilepsy is not often studied, especially in resource-poor countries, as Brazil, where superstitions, negative attitudes and lack of knowledge impair the relation between the community and people with epilepsy. Purpose: This article has the objective to introduce important aspects of stigma in epilepsy: conceptualization and models of stigma in the medical and social area; stigma and quality of life; neurobiological aspects and strategies to deal with it. Conclusions: This paper provides an overview of the stigma, including its different aspects. Stigma is a multifactorial concept and for this reason, to combat stigma it requires a broad intervention, involving medical, psychological and social areas. The understanding of the process of stigma contributes to a change of the social interpretation of the epilepsy, in a direction of a construction of a society more tolerant, where the differences are respected.
\end{abstract}

Key words: stigma, quality of life, epilepsy.

\footnotetext{
* Psicóloga. Doutora em Neurociências, Departamento de Neurologia da Faculdade de Ciencias Médicas da UNICAMP. Presidente da ASPE

** Médico. Doutor em Neurociências pela McGill University (Canadá). Professor associado do Departamento de Neurologia da FCM/UNICAMP.

Presidente fundador da ASPE. Departamento de Psicologia - Assistência à Saúde de Pacientes com Epilepsia - www.aspebrasil.org Received Aug 01, 2006; accepted Oct 06, 2006.
} 


\section{INTRODUÇÃO}

Estigma é um conceito que vem sendo estudado há muitos anos. Na literatura, o conceito de estigma vem sendo aplicado em diferentes situações, como hanseníase ${ }^{(1)}$, câncer ${ }^{(2)}$ e doenças mentais ${ }^{(3,4)}$. Até hoje, o termo estigma é usado para definir uma característica diferente do "normal" e é comumente associada a doenças e condições médicas ${ }^{(5)}$.

Muitas condições sofrem com o estigma, pois além de ser necessário lidar com os sintomas da doença, é preciso considerar as crenças e mitos que a cercam e que resultam na perpetuação do estigma na sociedade ${ }^{(6)}$.

$\mathrm{Na}$ neurologia e, especialmente na epilepsia, o preconceito é muito grande e muitas vezes, é considerado mais estressante e prejudicial do que a própria condição em $\mathrm{si}^{(7,8)}$. O rótulo de ser "epiléptico", muitas vezes associado à "personalidade epiléptica" leva à atenção pública, que por sua vez, conduz à atitudes de discriminação ${ }^{(9,10)}$.

\section{O QUE É ESTIGMA?}

Em muitas situações, a definição de estigma é parecida com a existente nos dicionários ou com aspectos relacionados a estereótipos negativos ou rejeição ${ }^{(3)}$. Na língua portuguesa, segundo o dicionário Aurélio ${ }^{(11)}$, a palavra estigma é definida como "cicatriz, sinal". Com o passar dos anos e o desenvolvimento dos estudos, a palavra estigma começou a ser relacionada com degradação e muitos pesquisadores começaram a estudá-la.

Nas doenças mentais, o estigma provém do medo do desconhecido e de falsas crenças que originam a falta de conhecimento e a não compreensão da situação. O estigma nestes casos isola o indivíduo em relação aos outros, como se fosse uma pessoa marcada por antigas crenças da doença. Por causa desta discriminação, quando em contato com novas oportunidades de relacionamentos ou de emprego, a pessoa que tem ou teve uma doença mental se esconde atrás de um "disfarce", de modo a manter sua identidade secreta. Este disfarce é resultado do medo de ser rejeitado ou desvalorizado devido a uma doença, como se esta fosse um mal ${ }^{(12,13)}$. Além das doenças mentais, a homossexualidade e o câncer eram vistas também como condições extremamente estigmatizantes ${ }^{(14)}$.

$\mathrm{Na}$ antiguidade clássica, os gregos criaram o termo estigma para fazer referência a sinais corporais extraordinários e ruins. A presença do estigma era característica de uma pessoa marcada, ritualmente poluída e que devia ser evitada. Na era Cristã, o estigma foi dividido em dois níveis: um de natureza sagrada, sendo que o estigma era sinal corporal de graça divina e o outro, uma alusão médica de distúrbio físico ${ }^{(15)}$.

Goffman $^{(15)}$ foi quem introduziu o conceito de estigma mais usado até hoje. Segundo ele, o estigma é definido como referência a um atributo depreciativo, fraqueza ou desvantagem. Em outras palavras, a pessoa estigmatizada é considerada como tendo uma característica diferente da aceita pela sociedade e é tratada de maneira diferente pela comunidade, que mostra conceitos errados e preconceituosos sobre o indivíduo. Para Goffman ${ }^{(15)}$, existem três tipos de estigma: 1. anormalidades do corpo (deformidades físicas), 2. culpas de caráter individual (crenças falsas e rígidas, alcoolismo, homossexualidade, desemprego, vícios), 3. estigmas tribais de raça, nação e religião.

Becker ${ }^{(16)}$ deu continuidade a este conceito ressaltando que os grupos sociais criam normas, cujas infrações constituem desvios e as pessoas que infringem estas normas são rotuladas como diferentes das demais. De acordo com seu ponto de vista, o estigma não é característica da atitude da pessoa, mas uma consequência da aplicação de normas sociais.

Anos mais tarde, Jones e colaboradores ${ }^{(17)}$, a partir da observação do estudo de Goffman, falam que o estigma é a relação existente entre o atributo e o estereótipo, ou seja, é uma característica (atributo) que aproxima a pessoa de características indesejáveis (estereótipo).

Pouco depois, Stafford e Scott ${ }^{(18)}$ definiram o estigma como sendo uma característica da pessoa contrária à norma social. Crocker e colaboradores ${ }^{(19)}$ sugeriram que a pessoa estigmatizada possui, ou acredita que possui, um atributo que é desvalorizado em contextos sociais particulares.

Tempos mais tarde, Reingold ${ }^{(20)}$ ampliou os tipos de estigma propostos por Goffman para cinco grupos: 1. comportamentos (abuso de álcool e drogas, homossexualidade, abuso sexual), 2 . anormalidades estruturais (problemas faciais, pigmentação da pele - vitiligo, problemas corporais - obesidade), 3. anormalidades funcionais (físicas, motoras, mentais, de linguagem, de audição e outras epilepsia), 4. doenças contagiosas (AIDS, tuberculose, doenças sexualmente transmissíveis), 5. outros (câncer).

Uma das razões pelo fato do termo estigma possuir uma infinidade de definições é o uso em inúmeras condições, sendo que cada definição diz respeito à características específicas de cada situação ou condição em particular. Outro motivo da variabilidade de conceitos sobre estigma pode residir no fato de que o estudo do estigma é multidisciplinar, com contribuições de diferentes áreas, como psicologia, sociologia, antropologia, entre outras. Desta maneira, os estudiosos do tema dão suas definições de acordo com suas orientações teóricas, o que pode resultar em diferentes visões sobre o mesmo tema ${ }^{(3)}$.

Existem inúmeras implicações para uma pessoa que se sente estigmatizada. Segundo Ryan et al.(21), o estigma está no valor dado por outras pessoas perante uma situação e para muitas delas, o estigma é um "companheiro diário"(14). Com isso, são afetados vários aspectos pessoais 
do indivíduo, como auto-estima, autoconfiança e qualidade de vida ${ }^{(14,22)}$.

\section{MODELOS DE ESTIGMA}

Na literatura, existem vários modelos para a explicação do estigma. Alguns dão ênfase à maneira de perceber o estigma ou aos tipos existentes, outros enfatizam as etapas em um processo de estigma. O mais importante é analisar estes modelos e aproveitar os pontos positivos de cada um deles.

O primeiro deles, desenvolvido por Goffman ${ }^{(15)}$, distingue dois tipos de estigma: estigma visível e estigma invisível. O visível é percebido ou sentido pelas pessoas que possuem uma característica aparente e, por isso, a maior preocupação é centrada na maneira de lidar com a tensão gerada na interação com a sociedade. O estigma invisível só se torna real quando a característica é revelada, direta ou indiretamente. Dessa forma, surge um outro problema: o controle da informação de sua própria condição. Por isso, em muitas condições, as pessoas escondem sua doença por vergonha ou medo de serem julgadas ou estigmatizadas ${ }^{(9,14,23,24)}$.

Dois grupos de trabalho, Link \& Phelan ${ }^{(3)}$ e Jones et al. (1984), formularam modelos alternativos para o estigma que, apesar de possuírem passos diferentes, representam um mesmo paradigma no processo do estigma.

Jones e colaboradores ${ }^{(17)}$, em seu modelo de estigma, propuseram seis dimensões: 1 . o quanto a característica estigmatizante é visível para a sociedade; 2. duração desta característica; 3. o quanto interfere nas relações interpessoais do indivíduo; 4. reações subjetivas frente à característica estigmatizante; 5 . a origem da característica (acidente, doença, etc) e 6. o perigo que esta condição pode causar para outras pessoas.

Para Link \& Phelan ${ }^{(3)}$, o estigma envolve cinco processos sociais. No primeiro, as pessoas distinguem e rotulam diferenças nas pessoas. Segundo, as crenças das culturas dominantes relacionam as características indesejáveis à estereótipos negativos e as pessoas começam a perceber que são "diferentes". No terceiro processo social, as pessoas rotuladas são distribuídas em categorias distintas e conseqüentemente, separadas das outras. No quarto processo, as pessoas rotuladas vivenciam a perda de status social e a discriminação. E o quinto processo é a força do estigma, como sendo a produção social do estigma.

Além destes modelos, Ablon ${ }^{(14)}$ propôs cinco dimensões para se entender melhor o processo do estigma nas condições de saúde: natureza da condição, fontes que criam e perpetuam o estigma, natureza das populações estigmatizadas, tratamentos e estratégias de enfrentamento. A natureza da condição refere-se à compreensão da condição em si, ou seja, qual a história da mesma, quais suas características para o estigma. As fontes que criam ou perpetuam o estigma envolvem atitudes e regras familiares, escolares e sociais. A natureza da população estigmatizada enfoca o tipo de sociedade em que vivem estas populações, pois pessoas privadas de benefícios e serviços de saúde tendem a sentir maior estigma. Os tipos de tratamento envolvem o entendimento dos que são realmente benéficos para o paciente, na medida em que existem alguns tratamentos ilegais, que podem aumentar os conflitos para o mesmo. E por último, as estratégias de enfretamento dizem respeito à maneira pela qual os indivíduos estigmatizados lidam com o estigma e com as dificuldades diárias.

\section{ESTIGMA NA EPILEPSIA}

Epilepsia é uma condição associada com significativas conseqüências psico-sociais e o fator central é sua natureza estigmatizante ${ }^{(25)}$. É considerada por Ablon ${ }^{(14)}$ uma das doenças crônicas com maior nível de estigma, sendo que os pacientes relatam o estigma como uma das grandes dificuldades que vivenciam com a epilepsia ${ }^{(26)}$. O estigma na epilepsia inicia-se já com a origem de seu nome, pois epilepsia é uma palavra de origem grega que significa ser invadido, dominado ou possuído. Com isso, podemos ter uma explicação mágica, sobrenatural, associada com possessão por espíritos divinos ou malignos ${ }^{(27)}$.

Registros da antiguidade mostram que o estigma na epilepsia não é uma questão recente. Já nos anos 2000 a.C., as pedras babilônicas possuíam descrições de algumas manifestações clínicas que hoje podem ser caracterizadas como crises epilépticas ${ }^{(28)}$. Desde aquela época, o preconceito existia: os escravos podiam ser devolvidos e contrato rescindido se a pessoa apresentasse crises no prazo de um mês depois de sua contratação ${ }^{29}$. Até 1970, em alguns países do mundo, como Reino Unido, as pessoas com epilepsia eram proibidas de se casarem ${ }^{22}$.

Na Bíblia, também aparecem passagens que falam da epilepsia. Em São Marcos, capítulo IX, versículos 13 a 28, aparece uma parábola onde Jesus expulsa o demônio do corpo de um menino, que apresentava convulsões, com prece e orações ${ }^{(30)}$.

Além disso, a epilepsia também foi retratada em pinturas. Apesar de haver contestações, em uma obra do Vaticano, há uma obra, chamada "Transfiguração de Rafael”, que retrata um menino em uma postura que representa uma crise epiléptica ${ }^{(27)}$.

Historicamente, a epilepsia tem sido conceituada como diferente e esta diferença envolve a aplicação de normas e regras contra as pessoas rotuladas "epilépticas"(31). O diagnóstico inicial da epilepsia traz dúvidas sobre as crises, as drogas anti-epilépticas e o medo da reação dos outros. Além disso, as pessoas possuem suas próprias crenças 
sobre a condição, o que pode contribuir para as atitudes de estigma ${ }^{(32)}$.

$\mathrm{Na}$ epilepsia, o estigma tem sido considerado um dos mais significativos fatores que influenciam negativamente a vida diária do paciente e sua família ${ }^{(31,33,34)}$. Na epilepsia, o estigma afeta as pessoas de diferentes maneiras, podendo influenciar as relações sociais, as oportunidades escolares e de emprego e os aspectos emocionais ${ }^{(32,35,36)}$.

Alguns autores ${ }^{15}$ falam que a epilepsia é uma condição estigmatizante, pois as pessoas não se adequam às normas sociais devido às crises imprevisíveis, muitas vezes de natureza dramática. Com isso, a sociedade tem medo de lidar com uma pessoa tendo crise epiléptica.

Em 1972, Bagley (37) dizia que a persistência das teorias que consideram a epilepsia como maléfica e estigmatizante são baseadas no "terror anônimo", no qual as pessoas com crise fazem exatamente o que outras temem fazer: perder o controle e retroceder a um estado mais primitivo de existência.

Anos mais tarde, Ablon ${ }^{(14)}$ complementa os achados de Goffman, dizendo que os sintomas da epilepsia afetam os valores da sociedade, pois estes prezam o controle e a previsibilidade das situações, o que não ocorre na epilepsia. Diiorio et al. ${ }^{(5)}$ acrescentam que a epilepsia é uma condição propensa ao estigma por causa do não controle das crises, o que gera medo e inquietação.

Devido ao caráter inesperado das crises, a epilepsia sempre possuiu um caráter místico, dependente de interferências sobrenaturais, sendo conhecida como o "mal sagrado". Por causa de sua incerteza clínica e do seu significado social, o impacto da epilepsia da vida das pessoas é muito significativo ${ }^{(38)}$.

Desde o diagnóstico da epilepsia, aparecem mudanças mudança na percepção e no grau de controle da pessoa. A partir dele, surgem dúvidas e questionamentos, que quando não respondidos, elevam a ansiedade e o stress, propiciando medo e confusão. Aparece o medo de ser diferente, a preocupação quanto ao futuro, acompanhados da dificuldade para se inserir na comunidade, para conseguir um emprego ou formar uma família, causando problemas no bem-estar e na autonomia da pessoa ${ }^{(9,39)}$.

De acordo com Hermann \& Whitman ${ }^{(40)}$, existem três grupos de variáveis que influenciam este impacto da epilepsia na vida do indivíduo: 1 . variáveis da epilepsia (idade de início, grau de controle das crises, duração e tipo da crise, etiologia); 2 variáveis medicamentosas (tipo e dosagem da medicação anti-epiléptica, nível sérico); 3. variáveis psico-sociais (medo das crises, estigma percebido e senso de discriminação, grau de ajustamento ao diagnóstico, suporte social). Separar estes três grupos é um pouco difícil, mas ter uma noção dos mesmos auxilia no entendimento do impacto geral desta condição na vida diária da pessoa com epilepsia(41).
Com isso, existe uma percepção negativa da epilepsia que, junto com o estigma associado, é legitimada pelas pessoas com epilepsia ${ }^{22,42}$. A epilepsia passa a significar perdas em diferentes áreas, tais como: física, saúde, emprego, relações sociais e familiares. Auto-estima e autoconfiança também são afetadas, contribuindo para uma diminuição da qualidade de vida. Quando a pessoa se vê como "epiléptica", um mundo de significados e crenças é ativado, influenciando negativamente seu ajustamento psico-social. Como resultado, a não aceitação ou a rejeição do diagnóstico de epilepsia passa a ser a maneira mais simples e rápida encontrada pelos pacientes neste primeiro momento. $\mathrm{O}$ medo e a vergonha passam a ser comuns na convivência diária com a epilepsia. Por causa da percepção do preconceito, os pacientes com epilepsia, na tentativa de se sentirem "normais", escondem seu diagnóstico e sua condição. Por sentirem sua identidade ameaçada, encontram formas de controlar a informação sobre sua condição: ou escondem o que tem ou usam termos mais suaves para a descreverem. O segredo passa a ser peça fundamental na adaptação destas pessoas, e o esforço que fazem para manter esta informação em segredo é proporcional à intensidade do estigma percebido ${ }^{(24)}$.

Além disso, para uma pessoa sentir-se estigmatizada é necessário que ela acredite nas idéias inadequadas da comunidade. Muitas pessoas não se sentem mais capazes para lidar com esta situação, o que produz alterações comportamentais (vergonha, insegurança, isolamento) e maiores dificuldades de ajustamento psico-social ${ }^{(9,22,31,32,43)}$. A partir daí, desenvolvem características negativas, que acreditam ser reais e que, segundo Scambler \& Hopkins ${ }^{(9)}$, reduzem as oportunidades. Tudo isso influencia negativamente sua qualidade de vida e reforça o estigma existente, gerando possibilidades maiores de ansiedade e depressão e baixos níveis de auto-estima e autoconfiança ${ }^{(41,44-46)}$.

Além disso, ainda nos dias de hoje, é comum encontrarmos lacunas no conhecimento sobre epilepsia. Alguns pacientes ainda acreditam que as crises representam um tipo de maldição ou aflição espiritual. Outros parecem ter sensações diferentes durante as crises que ocultam para seus médicos e outros profissionais envolvidos, por causa do receio de serem considerados "loucos"(47,48). Com estas informações inadequadas, aparecem as atitudes negativas perante a epilepsia, que são tidas como um fenômeno comum no mundo todo, sendo considerado o maior causador de discriminação social contra as pessoas com epilep$\operatorname{sia}^{(14,14,49)}$.

Devido ao preconceito e às atitudes negativas, as pessoas com epilepsia tendem a enfrentar problemas psicosociais, como medo, vergonha, isolamento social, dificuldades nos relacionamentos sociais, restrição de atividades, entre outros. A partir daí, o paciente deixa de se inserir na sociedade, pois não consegue um emprego, não se sente 
aceito e tem dificuldades para formar uma família. Por esta razão, além de exigir uma adaptação a um novo estilo de vida, a epilepsia também exige uma redefinição de identidade das pessoas. Porém, em cada fase da vida, a epilepsia afeta diferentes aspectos.

Quando a epilepsia tem seu início na infância, o estigma é desencadeado já na primeira crise. Com o diagnóstico da epilepsia, na maioria das vezes, os pais apresentam sentimentos de ansiedade, culpa e tristeza ${ }^{(50)}$. Estes sentimentos fazem com que os pais se comportem de maneira inapropriada, exibindo superproteção, permissividade excessiva ou rejeição e baixa expectativa. Os pais parecem tratar as crianças como doentes, achando que qualquer atividade pode precipitar uma crise ${ }^{(51)}$. A partir destas reações dos pais, as crianças consequentemente aprendem que há algo de errado com elas e apresentam comportamentos de dependência, insegurança, irritação e imaturidade. Dessa maneira, o estigma é perpetuado na família, fazendo com que tenha menor qualidade de vida e mais restrições de atividades e de comunicação ${ }^{(52)}$. É importante ressaltar que a maneira com que os pais reagem à epilepsia forma a base de como as crianças vão interpretar a epilepsia e se relacionar com outras pessoas, influenciando a dinâmica familiar e o ajustamento psico-social( ${ }^{(53)}$. A escola pode ser considerada também um outro tipo de dificuldade para a criança, pois o início da vida escolar significa um evento significativo, representado o primeiro contato com as interações sociais, normas e valores. Entretanto, se a criança é tratada como diferente e se sente como tal, podem aparecer os primeiros problemas nas relações interpessoais ou até no desempenho acadêmico ${ }^{(14)}$.

$\mathrm{Na}$ adolescência, por ser uma fase de mudanças e questionamentos, as principais dificuldades parecem estar relacionadas ao prognóstico da epilepsia, por existir o desejo de independência e de autonomia dos jovens. A epilepsia começa a afetar diferentes áreas: estudos, relacionamentos sociais, possibilidade de dirigir e de tomar bebidas alcoólicas, sexualidade, restrições de lazer, entre outros $^{(54)}$. Além disso, existe o receio da crise ocorrer em público por medo da consequente exclusão social. A autonomia se contrapõe à imprevisibilidade das crises, o desejo de agrupamento ao preconceito existente em nossa sociedade. Com todas estas incertezas, o adolescente tem sua auto-estima e auto-confiança abaladas, pois começa a se achar diferente de outras pessoas, o que limita suas oportunidades de crescimento pessoal e profissional.

Quando adultas, as pessoas estão mais estabilizadas em suas profissões e estilos de vida e, por isso, a epilepsia desencadeia implicações negativas nas relações sociais e no emprego, gerando conflitos familiares, sociais e econômi$\cos ^{(36)}$. Altas taxas de desemprego ou subemprego parecem contingentes às situações de discriminação. Baixos índices de casamento podem ser explicados pelo limitado contato social, associado ao medo da rejeição. A baixa auto-estima e a pobre qualidade de vida dos adultos com epilepsia parece ser resultado da percepção do estigma e das dificuldades no trabalho e nos relacionamentos ${ }^{(55)}$. Além disso, quando as pessoas com epilepsia já estão no papel de pais, muitas vezes se sentem envergonhados e incapacitados em lidar com as crianças, por apresentarem crises diante delas.

Diante do exposto, podemos dizer que o estigma na epilepsia é alterado de acordo com a situação no qual está inserido. Na área familiar, estão operando fatores relacionados à maneira como cuidar da pessoa com epilepsia; na área médica, o tratamento é o diferencial, sendo que não é esperado que exista falta de conhecimento; na área social, o medo da crise em público chama atenção, especialmente devido ao desconhecimento existente.

Um importante aspecto merece destaque com relação ao estigma na epilepsia. Um estudo realizado em Campinas ${ }^{(56)}$ mostrou que existe uma diferença na percepção do estigma quando utilizamos diferentes tipos de linguagem, no caso "pessoas com epilepsia" e "epilépticos". As respostas mostram que a comunidade possui uma percepção mais positiva com relação à epilepsia quando se fala em pessoas e não em rótulos diretos. Com isso, podemos afirmar que a maneira como nos referimos aos pacientes com epilepsia pode influenciar nossos comportamentos perante às mesmas. $\mathrm{O}$ uso de rótulos sociais esconde a verdadeira identidade da pessoa e contribui ainda mais para as dificuldades sociais e emocionais das pessoas com epilepsia. Estudos realizados em diferentes locais do mundo mostram a importância de fatores sociais no entendimento do estigma, em especial os relacionamentos familiares e sociais, os aspectos emocionais, o trabalho e os planos para o futuro $^{(22,38,57)}$.

Uma das maneiras de se explicar as diferenças na percepção do estigma leva em conta o modelo de estigma na epilepsia como um processo contínuo de elementos interligados ${ }^{(58)}$. Com base nos modelos já propostos por diferentes pesquisadores da área, elaboramos um modelo próprio de estigma na epilepsia no qual o estigma inicia-se com uma característica, que pode ser visível ou invisível. Com isso, aparece a percepção subjetiva que cada pessoa tem da condição e da característica estigmatizante, que depende de experiências pessoais e história de vida. A partir desta percepção, as pessoas interagem com o ambiente, percebendo se esta característica interfere nestas relações sociais. Quando a pessoa se coloca no mundo, fica sujeita à discriminar ou à discriminação e com isso, começa a reagir a partir de suas experiências pessoais e história de vida. De maneira simples, podemos perceber neste modelo que o estigma na epilepsia é um processo que envolve cinco passos distintos, mas interligados. A partir da percepção da característica diferente, que é dependente das expe- 
riências pessoais e de aprendizagem, a pessoa tem uma maneira específica de perceber o estigma e de lidar com o mesmo.

A partir deste modelo, foi feito um estudo sobre a percepção de estigma na epilepsia na cidade de Campinas, como parte integrante do Projeto Demonstrativo Brasileiro da Campanha Global "Epilepsia fora das Sombras"(59), executado pela ASPE (Assistência à Saúde de Pacientes com Epilepsia). Neste estudo ${ }^{(58)}$, realizamos uma pesquisa epidemiológica na cidade de Campinas, SP (população aproximada: 1 milhão de habitantes). $O$ instrumento utilizado foi a Escala de Estigma na Epilepsia (EEE) ${ }^{60,61}$ que contém 24 itens sobre a percepção do estigma na sociedade. Os resultados deste estudo, envolvendo 1.850 sujeitos mostraram que a magnitude da percepção do estigma na epilepsia varia de acordo com diferentes segmentos da sociedade, enfatizando que aspectos sócio-culturais como sexo, religião e nível de escolaridade são importantes fatores operantes de estigma.

\section{ESTIGMA E QUALIDADE DE VIDA NA EPILEPSIA}

A epilepsia possui muitas facetas que diferem de pessoa para pessoa e que acabam na maioria das vezes comprometendo a qualidade de vida dos pacientes com epilepsia e suas famílias ${ }^{(52,62-64)}$.

Antigamente, os profissionais da área da saúde não se preocupavam com os aspectos da qualidade de vida de seus pacientes e sim, com os seus sintomas. Com o passar dos anos e a evolução do conhecimento médico e social, passou a ser considerado também o impacto da doença em todos os aspectos da vida do paciente, ou seja, a qualidade de vida passou a ser um dos fatores influenciados pela doença $^{(55,65)}$.

Para se chegar a uma definição mais satisfatória de qualidade de vida, algumas idéias foram sugeridas para a uniformização e melhor definição do conceito, como estado subjetivo de saúde e de estado funcional ${ }^{(66)}$. Depois de vários estudos, a Qualidade de Vida (QV) foi definida pela Organização Mundial de Saúde como "a percepção do indivíduo de sua posição na vida, no contexto da cultura e sistema de valores nos quais ele vive e em relação aos seus objetivos, expectativas, padrões e preocupações"(67). Para contribuir para uma melhor definição de qualidade de vida, Hornquist ${ }^{68}$ ressaltou a satisfação das necessidades humanas como um dos principais fundamentos para a qualidade de vida, sendo que a mesma poderia ser definida em termos de grau de satisfação das necessidades física, psicológica, social, marital e estrutural. Calman ${ }^{(69)}$ acrescentou que a diferença entre as experiências reais e as expectativas da pessoa define a qualidade de vida e Cramer ${ }^{(70)}$ enfatizou o equilíbrio entre o bem-estar percebido e o desejado.
$\mathrm{Na}$ área da saúde, especialmente da epilepsia, a qualidade de vida refere-se à percepção individual do bem-estar físico, psicológico e social do indivíduo. É um conceito composto de três dimensões: física, mental e social. A dimensão física diz respeito ao estado de saúde geral, considerando a freqüência e intensidade das crises, os efeitos colaterais dos medicamentos e as tarefas de vida diária. A dimensão referente aos aspectos mentais é caracterizada pela percepção do estigma e do preconceito, condição emocional, auto-estima, transtornos associados (depressão, ansiedade) e cognição. Os aspectos sociais da qualidade de vida referem-se às atividades sociais no âmbito da família, do trabalho e dos amigos ${ }^{(71)}$.

Muito se tem falado sobre os efeitos da qualidade de vida nas pessoas com epilepsia e suas famílias ${ }^{(7,22)}$. Apesar de ser um conceito utilizado há bastante tempo, atualmente ganhou maior importância na prática clínica de condições crônicas ${ }^{(71)}$. Estudos têm mostrado que a epilepsia influencia a qualidade de vida dos pacientes, pois estas pessoas possuem menores chances de casar e ter filhos ${ }^{(72)}$ e de conseguir ou manter um emprego ${ }^{(36)}$.

Blummer \& Herman ${ }^{(73)}$ citam os seguintes fatores que influenciam na qualidade de vida na epilepsia: percepção do estigma e da discriminação, ajustamento à epilepsia, estado ocupacional, medo das crises, preocupação financeira, mudanças de vida, apoio social e lócus de controle. Além disso, vários estudos têm enfatizado a relação entre estigma e qualidade de vida. Desde a década de 70 observa-se que o estigma tem uma relação positiva com tristeza, depressão, ansiedade e sintomas somáticos e uma relação negativa com auto-estima e qualidade de vida.

Schneider \& Conrad $^{(24)}$ e Scambler \& Hopkins ${ }^{(26)}$ identificaram o impacto da epilepsia nas atividades de trabalho e escola, casamento e vida familiar. Anos mais tarde, Jacoby ${ }^{(74)}$ identificou que pacientes que se sentem estigmatizados possuem maior preocupação sobre sua condição e seu futuro e baixos níveis de auto-estima e autonomia, influenciando assim sua qualidade de vida. Anos depois, foram evidenciadas algumas variáveis psicológicas que influenciam a qualidade de vida da pessoa com epilepsia, as quais podemos destacar solidão, ajustamento psico-social, estratégias de enfrentamento e percepção de estigma $^{(7)}$.

Recentemente, Morrell( ${ }^{(32)}$ enfatizou a existência de uma relação negativa entre estigma e qualidade de vida, ou seja, quanto maior o estigma existente, menor a qualidade de vida e vice-versa. Segundo ela, os pacientes enfrentam problemas em algumas atividades, como trabalhar e dirigir, que os legitimam socialmente e são essenciais para a vida urbana. Por isso, é importante avaliar o impacto da epilepsia na qualidade de vida destes pacientes para que as barreiras do preconceito e do estigma sejam diminuídas. 
Jacoby ${ }^{(22)}$ ressaltou a interferência da epilepsia na qualidade de vida das pessoas, uma vez que a família toda sente o estigma, pois as dificuldades são espalhadas, comprometendo a qualidade de vida não apenas do paciente, mas da família como um todo. Além disso, ela salienta a relação entre estigma e qualidade de vida, no sentido de que crenças e desinformação contribuem para a lacuna de tratamento e conseqüentemente, para a diminuição da qualidade de vida e para o aumento do estigma associado.

Todos estes dados fornecem uma evidência da maneira pela qual o estigma influencia a vida das pessoas com epilepsia e sugere possíveis correlações entre estigma e qualidade de vida. Dessa maneira, considerar estes dois aspectos na vida do paciente com epilepsia e da comunidade em que ele vive, reforça a necessidade de se ter uma visão global do indivíduo, levando-se em consideração não apenas seus sintomas físicos, mas também os aspectos psicológicos, incluindo o impacto da condição na vida do paciente e da sociedade.

\section{FATORES DESENCADEANTES E OPERANTES DO ESTIGMA NA EPILEPSIA}

Por ser um conceito multifatorial, os fatores desencadeantes ou etiológicos do estigma também são dependentes de múltiplos fatores. A falta de informação aliada à imprevisibilidade das crises e sua incontrolabilidade, a incerteza quanto ao prognóstico e o impacto social são importantes aspectos na determinação o estigma na epilepsia( ${ }^{(31,75)}$. Além disso, características pessoais também podem afetar, direta ou indiretamente, as respostas nas situações de discriminação ${ }^{(24)}$. Baker ${ }^{(76)}$ e Morrel $^{(32)}$ sugerem que o estigma tem forte relação com o aprendizado social e com o diagnóstico de epilepsia, além da frequência e severidade das crises. Outros fatores importantes que conduzem ao estigma na epilepsia: desconhecimento sobre a condição, pressão da mídia, reação dos familiares (atitudes negativas) frente ao diagnóstico, generalização das experiências de medo em outras situações ${ }^{(22)}$.

A falta de informação ${ }^{(14,22,31)}$ está presente em grande parte das pessoas, especialmente no que se refere à definição de epilepsia, suas causas, os tipos de tratamento existentes e os procedimentos durante a crise. Por esta razão pode ser considerada como um dos fatores operantes na perpetuação do estigma na sociedade, como também observado por outros autores, em seus respectivos países ${ }^{(22)}$.

Esta desinformação da população, ainda presente nos dias atuais é reforçada por idéias e crenças inadequadas, provenientes do senso-comum, sem base científica. É comum ouvirmos equívocos sobre a epilepsia, fazendo com que algumas pessoas sintam-se mais prejudicadas com a consequência destas crenças do que com a própria condição em $\mathrm{si}^{(7)}$. Estas lacunas no conhecimento podem ser decorrentes de crenças relativas à epilepsia. Isso pode gerar comportamentos inapropriados, como vimos neste estudo, trazendo dificuldade nas relações sociais e aumentando os sentimentos de insegurança e preocupação, que podem conduzir ao estigma ${ }^{(52)}$. Geralmente, existe um alto nível de ansiedade alimentado por crenças irracionais e pelo fato da epilepsia vir associada a problemas de comportamento ou personalidade ${ }^{(51)}$.

As principais crenças na epilepsia referem-se a comportamentos durante a crise e consequências da epilepsia. O medo do paciente engolir a língua, que aparece na maioria dos sujeitos deste estudo, pode fazer com que muitas pessoas introduzam objetos (caneta, dedos, pano) para evitar que a língua se enrole ${ }^{(77)}$. Outra idéia comum é o receio da epilepsia vir acompanhada de doença ou retardo mental77. Estas idéias populares são errôneas, pouco empíricas e dificultam a obtenção das metas da pessoa; por isso, são chamadas de crenças irracionais. Crenças deste tipo, além de estarem baseadas na desinformação, podem interferir na qualidade de vida das pessoas, aumentado o estigma existente e dificultando seu ajustamento psicossocial $^{(53)}$.

Mas, é importante ressaltar que este aspecto não é o único fator operante de estigma, pois além da falta de conhecimento, existem comportamentos de discriminação (trabalho, escola, relacionamentos sociais, atividades de lazer) e sentimentos negativos (tristeza, fragilidade, inferioridade), que podem também estar relacionados à origem e/ou à perpetuação do estigma na sociedade.

Um aspecto que deve ser considerado é a relação do estigma com o trabalho, pois segundo vários autores, o desemprego e o subemprego são identificados como sérios problemas na epilepsia ${ }^{(36)}$. O trabalho é considerado como um dos principais problemas que os pacientes com epilepsia enfrentam no seu dia-a-dia ${ }^{(36,58)}$. Muitas pessoas são afastadas de seus cargos ou redirecionadas para outras áreas por causa da epilepsia. Os que permanecem no trabalho vivem com a ansiedade e o medo de perderem o empre$\mathrm{go}^{(36)}$. Isso acontece por motivos que variam desde a preocupação com a pessoa em crise e com os efeitos colaterais das medicações anti-epilépticas, até o alto nível de preconceito existente em nossa sociedade. Além disso, muito pacientes não conseguem emprego por terem tido menores chances de se qualificarem na escola ou em trabalhos informais. Neste contexto, é importante ressaltar que a formação escolar dos pacientes com epilepsia parece ser um problema. Desde o início da vida escolar, que representa os primeiros contatos com interações sociais e com futuras possibilidades de trabalho, a pessoa com epilepsia enfrenta dificuldades ${ }^{(14)}$. A crença de que crianças com epilepsia vão apresentar algum comprometimento intelectual ou até mesmo retardo mental parece colaborar com esta idéia ${ }^{(51)}$. 
Outro aspecto que merece destaque refere-se aos relacionamentos interpessoais. Estudos ${ }^{14,22,75}$ demonstram que as pessoas com epilepsia têm maior dificuldade de estabelecer contatos sociais e de formar uma família, o que é acentuado quando o estigma aparece. Na nossa experiência ${ }^{(58)}$, a grande maioria das pessoas com epilepsia, independente de outros fatores, como região onde vive, sexo, religião, classe social, diz que uma das principais dificuldades enfrentadas pelos pacientes está relacionada à convivência social, amizades ou namoro.

As atividades de lazer também são percebidas como problemas para as pessoas com epilepsia. Muitas vezes, os pacientes deixam de frequentar lugares sociais pelo receio de terem uma crise. Com isso, temos o chamado "estigma de cortesia" (15), através do qual as pessoas que convivem com os pacientes sofrem, muitas vezes, as mesmas privações dos mesmos. Neste sentido, percebemos o impacto socioeconômico da epilepsia. Os responsáveis deixam de trabalhar para tomar conta dos pacientes devido às crises ou às visitas regulares ao médico, interferindo assim na sua rotina diária de trabalho e, conseqüentemente, na sua renda familiar.

Familiares também evitam participar das atividades sociais por vergonha ou por superproteção. A vergonha ocorre pelo medo da reação de estigma dos outros e a superproteção, pelo cuidado excessivo à estes pacientes. Como as crises são inesperadas e ocorrem em freqüência imprevisível, a família geralmente utiliza comportamentos inapropriados, como a superproteção, a permissividade excessiva, para compensar a falta de controle que sente sobre as mesmas. Infelizmente, estas atitudes contribuem para problemas de ajustamento e de estresse familiar, contribuindo desfavoravelmente para o desenvolvimento adequado de toda a família(52).

\section{BASES NEUROBIOLÓGICAS DO ESTIGMA}

De acordo com os dados apresentados, podemos dizer que o estigma na epilepsia possui como principais fatores operantes as variáveis psicossociais. Porém, é difícil distinguir os fatores psicossociais dos neurobiológicos, uma vez que quando comparamos respostas de estigma na sociedade, percebemos uma ampla variedade de respostas que envolvem diferentes processos cognitivos de percepção e julgamento de uma característica considerada "anormal" pela sociedade. Neste contexto, agora vamos falar sobre as diferenças neurobiológicas e das redes neurais como um dos mais novos modelos para se explicar as diferentes percepções de estigma na epilepsia.

Freqüentemente, as pessoas julgam características e comportamentos dos outros. Este julgamento pode ser feito por crenças morais e por processos cognitivos e de aprendizagem ${ }^{(78,79)}$. Além disso, o funcionamento de redes neurais é envolvido em processos de percepção, como é o caso da percepção do estigma ${ }^{(80)}$. Pesquisas recentes ${ }^{(80,81)}$ enfatizaram o papel dos processos cognitivos, emocionais e intuitivos nas decisões humanas e na socialização. Com isso, podemos adicionar o contexto cultural, o processo e aprendizagem e o conhecimento social no processo do estigma.

Estudos com neuroimagem ${ }^{(79,81)}$ e evidências clínicas $^{(82)}$ enfatizaram as redes neurais envolvidas em alguns processos cognitivos, como o julgamento moral e o comportamento humano em geral. De maneira geral, os aspectos morais são resultados da integração entre o conhecimento social (córtex pré-frontal), conhecimento semântico (córtex temporal posterior) e estados motivacionais (sistema límbico). Nas habilidades sociais estão envolvidos: córtex órbito-frontal e amígdala ${ }^{(78)}$. Um outro estudo ${ }^{(83)}$ mostrou que o córtex pré-frontal e o sulco temporal são áreas responsáveis pela emoção e cognição social. Estes estudos mostram que existe uma associação entre regiões específicas do cérebro e funções específicas $^{(84)}$, sendo esse achado válido também para funções cognitivas complexas como o controle cognitivo das emoções ${ }^{(85)}$.

Estudos realizados na última década mostram que a ressonância magnética funcional (RMf) tem sido largamente utilizada na avaliação de funcionamento cerebral. Dentro das diferentes possibilidades técnicas, a mais utilizada é o contraste BOLD (Blood Oxygenation Level Dependent $)^{(86)}$, no qual, a atividade funcional do cérebro identificada é determinada pela subtração dos dois conjuntos de imagens (em atividade e em repouso) decorrente da diferença na concentração de desoxi-hemoglobina, resultante do metabolismo e fluxo sanguíneo ao córtex ativado ${ }^{(87)}$. Embora os mecanismos de acoplamento neurovascular e metabolismo sejam ainda objetos de pesquisa, aceita-se que o efeito BOLD tenha uma relação direta com a atividade neuronal ${ }^{(86,88)}$. Neste contexto, a RMf pode fornecer bases para estes processos cognitivos e emocionais. A integração de avaliação de aspectos psicossocial e neuroimagem permite explorar as bases neurais envolvidas na percepção do estigma na epilepsia. Com isso, podemos avaliar o mecanismo básico responsável pelo preconceito e estigma. Qual é o modelo básico de estigma na epilepsia? Por que, durante uma crise epiléptica, as pessoas sentem medo, dó ou tristeza? Qual é a o estímulo que desencadeia o processo do estigma?

Em um estudo piloto ${ }^{(89)}$ utilizando RMf, uma voluntária assistiu a um filme de uma crise epiléptica tônicoclônica e o resultado mostrou ativação em algumas áreas cerebrais, tais como: lobo parietal, áreas de Broadmann 9 (frontal inferior), 19 (occipital), 32 (giro do cíngulo - lobo límbico) e 37 (lobo temporal), além do giro frontal superior. Estes resultados apontam para algumas regiões des- 
critas no processamento de cenas de contexto emocional ou no controle cognitivo. Estudos deste tipo, que integram os aspectos psicossociais com a interpretação neurobiológica, proporcionam um entendimento mais amplo de processos emocionais e cognitivos na percepção do estigma na epilepsia. Porém, ações práticas devem ser direcionadas para que a epilepsia seja vista sob uma nova perspectiva. As estratégias podem abordar as diferentes fases do processo de estigma, desde a informação adequada até mudanças comportamentais, para combater seus efeitos negativos.

\section{ESTRATÉGIAS PARA SE REDUZIR O ESTIGMA NA EPILEPSIA}

De maneira prática, existem três principais formas de se trabalhar para conseguir a redução do estigma na epilepsia: 1. campanhas na mídia; 2. trabalho bio-psicossocial com pacientes e famílias; 3 . programas de educação continuada.

\section{Campanhas na mídia}

São as chamadas campanhas de desestigmatização ou de combate ao estigma.

Sabemos que informações sobre epilepsia são ferramentas fundamentais para reduzir o impacto do estigma na vida diária do paciente e seus familiares. Estudos sugerem que a informação correta é um importante fator na melhora das habilidades para lidar adequadamente com a epilepsia, através da diminuição do seu impacto social e psicológico e oferecendo o potencial para melhores relações sociais ${ }^{(90)}$. Porém, é importante ressaltar que apenas informações não bastam. É preciso direcionar as informações para alvos específicos ${ }^{(91)}$.

É preciso que campanhas na mídia de desestigmatização sejam feitas para mudar conhecimento e comportamentos sociais na epilepsia, pois algumas percepções e atitudes negativas podem ter sido induzidas por falsas crenças sobre epilepsia. Porém, o ideal é que estas campanhas sejam direcionadas para públicos específicos, para determinados segmentos da sociedade, considerando as diferenças de sexo, religião, escolaridade e conhecimento prévio sobre o tema.

\section{Trabalho bio-psicossocial com pacientes e famílias}

O trabalho bio-psicossocial envolve o atendimento integral da pessoa com epilepsia e sua família. Neste processo, é importante haver uma relação de troca entre as variáveis bio-psicossociais para, junto com os aspectos relacionados ao estigma na epilepsia, consigamos proporcionar uma atmosfera de entendimento, fazendo com que as pessoas consigam se inserir adequadamente na sociedade. Este trabalho consiste em: 1. atendimento médico adequado; 2. psicoterapia; 3. grupos de apoio ou grupos educativos.

\section{- Atendimento médico adequado}

O tratamento médico tem o objetivo de controlar as crises e com isso, a qualidade de vida das pessoas com epilepsia. Este tratamento deve considerar a prescrição adequada de medicação antiepiléptica e o controle dos efeitos colaterais das mesmas. A partir do momento em que o paciente passa a ter suas crises controladas, ele começa a se sentir melhor e, com isso, passa a se inserir na sociedade de maneira mais integral e plena.

\section{- Psicoterapia}

É um processo no qual a pessoa amplia a autoconsciência, aprendendo com seus sintomas e se desenvolvendo como pessoa. Na epilepsia, a psicoterapia visa a aceitação de si mesmo, a melhora da auto-estima e da autoconfiança, contribuindo assim para a melhora da qualidade de vida e redução do estigma associado.

\section{- Grupos de apoio ou grupos educativos}

São realizados com o objetivo de propiciar a inserção social da pessoa com epilepsia. Estes grupos podem ser realizados com profissionais da área da saúde e psicossocial, professores, comunidades, pacientes, familiares e pessoas em geral que se interessem por saber mais sobre epilepsia. Têm o objetivo de disseminar conhecimentos adequados sobre epilepsia, através da troca de experiências, permitindo às pessoas adquirir maior confiança no manejo da epilepsia, diminuindo assim o estigma associado ${ }^{(92)}$.

Com profissionais, o objetivo do trabalho em grupo é debater com os mesmos o que é epilepsia, quais são suas implicações práticas, suas causas e conseqüências, para que o paciente e sua família deixem de ser vítimas de preconceito e estigma na comunidade, contribuindo para seu fortalecimento ${ }^{(92)}$.

O grupo com familiares consolida a participação da família no processo de atenção ao paciente, pois ela está sofrendo os impactos secundários e generalizados da epilepsia. A família tem um papel mediador que favorece a recuperação do paciente, sendo que relações familiares saudáveis são ricas fontes de suporte social do paciente, sendo que quando os pais, cônjuges ou responsáveis têm maior conhecimento sobre a epilepsia e suas conseqüências, melhora a qualidade de vida da família como um todo ${ }^{(92)}$.

Os grupos de pacientes com epilepsia podem ser considerados um encontro de pessoas que compartilham da mesma problemática e, através dos mesmos, é criado um espaço que permite a troca de experiências e vivências. Estes grupos auxiliam no conhecimento de informações, na discussão de medos, ansiedade e confusões a respeito desta condição, sendo considerados uma oportunidade para compartilhar idéias, expressar sentimentos e trocar experiências. Além disso, as pessoas podem aumentar seus 
conhecimentos, discutir mágoas e desconfortos relacionados à epilepsia. É importante que as pessoas participem de grupos para obterem melhores informações sobre epilepsia e, consequentemente, diminuam o preconceito associado.

\section{Programas de Educação Continuada}

Estes programas visam a diminuição do estigma através da capacitação e educação continuada de profissionais da área da saúde ${ }^{(6)}$. Estes cursos são breves e proporcionam atitudes mais adequadas no manejo da epilepsia.

Em primeiro lugar, é importante ressaltar que os pacientes com epilepsia relatam que sua principal fonte de informação vem de profissionais da saúde e, muitos deles, falam que não recebem informação adequada ${ }^{(93)}$. Recentemente, alguns autores têm salientado a importância do conhecimento dos profissionais da saúde com relação à epilepsia ${ }^{(31,59)}$. Quando estes profissionais não têm informação adequada sobre determinada condição, há uma distorção na relação profissional-paciente, o que influencia a adesão ao tratamento ${ }^{(94)}$. E na epilepsia, isso é fundamental, pois sabemos que aproximadamente $70 \%$ dos casos são controlados quando o paciente segue o tratamento corretamente $^{(95,96)}$.

Os pacientes acreditam que os profissionais da saúde são as pessoas mais competentes para fornecer informação sobre qualquer condição clínica ${ }^{(94)}$ e, por isso, querem informações ${ }^{(97)}$. Por isso, a importância do conhecimento adequado destes profissionais no manejo integral aos pacientes com epilepsia.

Além disso, é necessário que professores sejam inseridos em programas deste tipo. Apesar de muitos já terem presenciado uma crise epiléptica, desinformação e mitos ainda persistem. A maioria deles não tem informações suficientes sobre a condição e suas conseqüências. Há também lacunas de conhecimento no que diz respeito à área educacional, como quociente intelectual ou receio da epilepsia estar acompanhada de retardo ou doença mental, já apontada por outras pesquisas ${ }^{(47,51)}$. Estes programas se fazem necessários uma vez que os professores são considerados modelos para as crianças, sendo importantes no desenvolvimento de idéias e de consciência crítica para seus alunos, exercendo uma importante influência sobre os mesmos.

Uma pesquisa feita com crianças de 10 anos de idade reforça a existência destes dados ${ }^{(98)}$, pois $56 \%$ das mesmas acreditam que epilepsia é uma doença que engole a língua, que é contagiosa ou que pode matar. Crianças nesta faixa etária possuem idéias consideradas como um reflexo das crenças sociais e da consciência coletiva sobre o preconceito na epilepsia, podendo conduzir à perpetuação do estigma ${ }^{(58)}$. As crianças entrevistadas na escola mostraram conhecimentos espontâneos, sem censura, mas infelizmen- te inadequados. Dizer que a epilepsia é uma doença que mata, que enrola a língua ou que é contagiosa reflete pelo menos em parte a atitude da sociedade perante esta condição. Isso porque as crianças verbalizam naturalmente o que aprendem, tanto na família, como na escola, mostrando que a sociedade em geral não tem conhecimentos adequados sobre a epilepsia, facilitando assim a perpetuação do estigma ${ }^{(23)}$.

Para isso, é fundamental haver uma política de saúde e educação que envolva capacitação, reciclagem e cursos de educação continuada. Se conseguirmos que estes profissionais, da área de saúde e educação, tenham maior informação sobre epilepsia, teremos melhor qualidade de vida para as pessoas com epilepsia.

\section{CONCLUSÃO}

Neste artigo, nós demos uma visão geral do estigma com seus diferentes aspectos. Pelo fato de ser um conceito multifatorial, o combate ao estigma requer também uma intervenção ampla, envolvendo as áreas médica, psicológica e social ${ }^{(5)}$. A partir das mudanças ocorridas na prática médica, com uma visão mais global no atendimento ao paciente, são considerados os aspectos psicológicos, sociais, comportamentais e familiares das doenças, além do aspecto físico. Com isso, conseguimos a retomada do modelo bio-psicossocial de saúde, que existe desde a época de Hipócrates. Este modelo considera, além dos sintomas biológicos da doença, o impacto exercido pelo estilo de vida e por variações pessoais e sociais sobre a saúde do indivíduo ${ }^{(65)}$.

A contribuição conjunta dos vários fatores na origem e na perpetuação do estigma na epilepsia é um passo importante para a mudança da interpretação social da epilepsia. Desta maneira, a sociedade pode mudar sua maneira de ver e de tratar as pessoas com epilepsia, conseguindo melhoras nas oportunidades de participação social e, conseqüentemente, na diminuição do impacto socio-econômico da epilepsia. Só assim, conseguiremos diminuir o estigma, construindo uma sociedade mais justa e tolerante, na qual as diferenças sejam respeitadas.

\section{REFERÊNCIAS}

1. Opala J, Boillot F. Leprosy among the Limba: illness and healing in the context of world view. Soc Sci Med. 1996; 42(1):3-19.

2. Fife BL, Wright ER. The dimensionality of stigma: a comparison of its impact on the self of persons with HIV/AIDS and cancer. J Health Soc Behav. 2000; 41(1):50-67.

3. Link BG. Stigma and its public health implications. Stigma and global health: developing a research agenda 2001.

4. Corrigan PW, Penn DL. Lessons from social psychology on discrediting psychiatric stigma. Am Psychol. 1999; 54(9):765-76.

5. DiIorio C, Osborne SP, Letz R, Henry T, Schomer DL, Yeager K. The association of stigma with self-management and perceptions of health care among adults with epilepsy. Epilepsy Behav. 2003; $4(3): 259-67$. 
6. Rusch N, Angermeyer MA, Corrigan PW. Mental illness stigma: concepts, consequences, and initiatives to reduce stigma. European Psychiatry. 2005; 20:529-39.

7. Suurmeijer TP, Reuvekamp MF, Aldenkamp BP. Social functioning, psychological functioning, and quality of life in epilepsy. Epilepsia. 2001; 42(9):1160-8.

8. Fisher RS. Epilepsy from the Patient's Perspective: Review of Results of a Community-Based Survey. Epilepsy Behav. 2000; 1(4):S9-S14.

9. Scambler G, Hopkins A. Generating a model of epileptic stigma: the role of qualitative analysis. Soc Sci Med. 1990; 30(11): 1187-94.

10. Baumann RJ, Wilson JF, Wiese HJ. Kentuckians' attitudes toward children with epilepsy. Epilepsia. 1995; 36(10):1003-8.

11. Ferreira ABH. Dicionário Aurélio da Língua Portuguesa. Rio de Janeiro: Editora Nova Fronteira; 1993.

12. ADEP. Associação de Apoio aos doentes depressivos e bipolares. [www.admd.pt] 2004. Ref Type: Electronic Citation.

13. Ballone GJ. Relatório sobre a saúde no mundo. OPAS-OMS-ONU, editor. [www.psiqueweb.med.br/acad/oms1.html] 2001. Ref Type: Electronic Citation.

14. Ablon J. The nature of stigma and medical conditions. Epilepsy Behav. 2002; 3(6S2):2-9.

15. Goffman E. Stigma: notes on the management of spoiled identity. New York: Touchstone; 1963.

16. Becker H. Outsiders: studies in sociology of deviance. New York: Free Press; 1963.

17. Jones E, Farina A, Hastof A, Markus H, Miller DT, Scott RA. Social Stigma: the psychology of marked relationships. New York: Freeman and Company; 1984.

18. Sttaford MC, Scott RR. Stigma deviance and social control: some conceptual issues. In: Ainlay SC, Becker G, Coleman LM, editors. The Dilemma of Difference. New York: Plenum; 1986.

19. Crocker J, Major B, Steele C. Social Stigma. In: Gilbert DT, Fiske ST, editors. The handbook of Social Psychology. Boston: Mc Graw Hill; 1998 . p.504-53.

20. Reingold AL. The study of stimatizing conditions: an epidemiologic perspective. Stigma and Global Health: developing a research agenda 2001. (Abstract)

21. Ryan R, Kempner K, Emlen AC. The stigma of epilepsy as a selfconcept. Epilepsia. 1980; 21(4):433-44.

22. Jacoby A. Stigma, epilepsy, and quality of life. Epilepsy Behav. 2002; $3(6 \mathrm{~S} 2): 10-20$.

23. MacLeod JS, Austin JK. Stigma in the lives of adolescents with epilepsy: a review of the literature. Epilepsy Behav. 2003; 4(2): 112-7.

24. Schneider JW, Conrad P. Having epilepsy: the experience and control of illness. Philadelphia: Temple University Press; 1983.

25. Baker GA, Brooks J, Buck D, Jacoby A. The stigma of epilepsy: a European perspective. Epilepsia. 2000; 41(1):98-104.

26. Scambler G, Hopkins A. Being epileptic: coming to terms with stigma. Sociol Health III. 1986; 8:26-43.

27. Temkim O. The falling sickness: a history of epilepsy from the Greeks to the beginnings of modern neurology. $2^{\text {nd }}$ ed. Baltimore: The Johns Hopkins University Press; 1971.

28. Wilson JV, Reynolds EH. Texts and documents. Translation and analysis of a cuneiform text forming part of a Babylonian treatise on epilepsy. Med Hist. 1990; 34(2):185-98.

29. Yacubian E. Epilepsia da antiguidade ao segundo milênio: saindo das sombras. São Paulo: Lemos Editorial; 2000.

30. Guerreiro CA, Li LM. Epilepsia: conceito e epidemiologia. São Paulo: Abbott; 2003. Ref Type: Catalog.

31. McLin WM, de Boer HM. Public perceptions about epilepsy. Epilepsia. 1995; 36(10):957-9.

32. Morrell MJ. Stigma and epilepsy. Epilepsy Behav. 2002; 3(6S2): 21-5.

33. Jacoby A, Chadwick D. Psychosocial problems in epilepsy. BMJ. 1992; 305(6845):117.
34. Collings JA. Epilepsy and well-being. Soc Sci Med. 1990; 31(2): $165-70$.

35. Jacoby A, Gorry J, Gamble C, Baker GA. Public knowledge, private grief: a study of public attitudes to epilepsy in the United Kingdom and implications for stigma. Epilepsia. 2004; 45(11):1405-15.

36. Salgado PC, Souza EA. [Impact of epilepsy at work: evaluation of quality of life]. Arq Neuropsiquiatr. 2002; 60(2-B):442-5.

37. Bagley C. Social prejudice and the adjustment of people with epilepsy. Epilepsia. 1972; 13:33-45.

38. Baker GA, Jacoby A, Buck D, Stalgis C, Monnet D. Quality of life of people with epilepsy: a European study. Epilepsia. 1997; 38(3):353-62.

39. Jacoby A, Buck D, Baker G, McNamee P, Graham-Jones S, Chadwick D. Uptake and costs of care for epilepsy: findings from a U.K. regional study. Epilepsia. 1998; 39(7):776-86.

40. Hermann B, Whitman S. Psychopatology in epilepsy: multietiological model. In: Whitman S, Hermann B, editors. Psychopatology in epilepsy: social dimensions. Oxford: Oxford University Press; 2001: 5-37.

41. Baker GA, Brooks J, Buck D, Jacoby A. The stigma of epilepsy: a European perspective. Epilepsia. 2000; 41(1):98-104.

42. Placencia M, Farmer PJ, Jumbo L, Sander JW, Shorvon SD. Levels of stigmatization of patients with previously untreated epilepsy in northern Ecuador. Neuroepidemiology. 1995; 14(3):147-54.

43. Jacoby A. Felt versus enacted stigma: a concept revisited. Evidence from a study of people with epilepsy in remission. Soc Sci Med. 1994; 38(2):269-74.

44. Baker GA. The psychosocial burden of epilepsy. Epilepsia. 2002; 43 Suppl 6:26-30.

45. Jacoby A. Epilepsy and the quality of everyday life. Findings from a study of people with well-controlled epilepsy. Soc Sci Med. 1992; 34(6):657-66.

46. Jacoby A. Assessing quality of life in patients with epilepsy. Pharmacoeconomics. 1996; 9(5):399-416.

47. Gumnit RJ. Living well with epilepsy. New York: Demos Vermande, 1997.

48. Devinsky O, Penry JK. Quality of life in epilepsy: the clinician's view. Epilepsia. 1993; 34(suppl 4):S4-S7.

49. Kim MK, Kim IK, Kim BC, Cho KH, Kim SJ, Moon JD. Positive trends of public attitudes toward epilepsy after public education campaign among rural korean residents. J Korean Med Sci. 2003; $18(2): 248-54$.

50. Lewis MA, Hatton CL, Salas I, Leake B, Chiofalo N. Impact of the Children's Epilepsy Program on parents. Epilepsia. 1991; 32(3): 365-74.

51. Thompson PJ, Upton D. The impact of chronic epilepsy on the family. Seizure. 1992; 1(1):43-8.

52. Fernandes PT, Souza EA. Identification of family variables in parents' groups of children with epilepsy. Arq Neuropsiquiatr. 2001; 59(4):854-8.

53. Fernandes PT, De Souza EA. [Simplified inventory of quality of life in childhood epilepsy: initial results]. Arq Neuropsiquiatr. 1999; $57(1): 40-3$.

54. Herranz JL. [Factors modifying treatment of adolescents with epilepsy]. Rev Neurol. 1999; 28(1):49-53.

55. Souza EA, Fernandes PT, Salgado PC, Doretto F. Mecanismos psicológicos e o estigma na epilepsia. Revista ComCiência. 2002; 34:98-103.

56. Fernandes PT, Li LM. Epileptic x Person with epilepsy: does it matter? Libro de Resumes/Abstracts Book; 2006. p.36. (Abstract)

57. Kleinman A, Wang WZ, Li SC, Cheng XM, Dai XY, Li KT et al. The social course of epilepsy: chronic illness as social experience in interior China. Soc Sci Med. 1995; 40(10):1319-30.

58. Fernandes PT, Li LM. Estigma na Epilepsia. Campinas: Departamento de Neurologia - FCM/UNICAMP; 2005.

59. Li LM, Sander JW. [National demonstration project on epilepsy in Brazil]. Arq Neuropsiquiatr 2003; 61(1):153-6. 
60. Fernandes PT, Salgado PC, Noronha ALA, Barbosa FD, Souza EA, Li LM. Stigma Scale of Epilepsy: conceptual issues. Journal of Epilepsy and Clinical Neuropshysiology. 2004; 10(4):213-8.

61. Fernandes PT, Salgado PC, Noronha ALA, Li LM. Stigma Scale of Epilepsy: epidemiologic study inCampinas. submitted 2005.

62. Reynolds EH. The ILAE/IBE/WHO Global Campaign against Epilepsy: Bringing Epilepsy "Out of the Shadows". Epilepsy Behav. 2000; 1(4):S3-S8.

63. Meinardi H, Scott RA, Reis R, Sander JW. The treatment gap in epilepsia: the current situation and way forwards. Epilepsia. 2001; 42:136-49.

64. Scott-Lennox J, Bryant-Comstock L, Lennox R, Baker GA. Reliability, validity and responsiveness of a revised scoring system for the Liverpool Seizure Severity Scale. Epilepsy Res. 2001; 44(1):53-63.

65. Guimarães SS. Psicologia da Saúde e doenças crônicas. In: Kerbauy RR, editor. Comportamento e saúde: explorando alternativas. São Paulo: ARBytes Editora; 1999. p.22-45.

66. Fitzpatrick R, Fletcher A, Gore S, Jones D, Spiegelhalter D, Cox D. Quality of life measures in health care. I: Applications and issues in assessment. BMJ 1992; $305(6861): 1074-7$.

67. WHO - World Health Organization. The first 10 years of the World Health Organization. 1958. Geneva. Ref Type: Catalog.

68. Hornquist JO. The concept of quality of life. Scand J Soc Med. 1982; 10:57-67.

69. Calman KC. Quality of Life in cancer patients and hypothesis. Journal of Medical Ethics. 1984; 10:124-7.

70. Cramer JA. Quality of life for people with epilepsy. Neurol Clin. 1994; 12(1):1-13

71. Guerreiro CA, Guerreiro MM. O paciente otimamente controlado. São Paulo; 1999.

72. Dansky LV, Andermann E, Andermann F. Marriage and fertility in epileptic patients. Epilepsia. 1980; 21:261-71.

73. Blummer DC, Hermann BP. Behavioral and emotional adjustment in epilepsy. In: Epilepsy Foundation of America, editor. Issues in epilepsy and quality of life. Landover; 1993.

74. Jacoby A. Assessing quality of life in patients with epilepsy. Pharmacoeconomics. 1996; 9(5):399-416.

75. Baker G. The Psychosocial Burden of Epilepsy. Epilepsia. 2002; 43 (suppl. 6):26-30.

76. Baker GA, Jacoby A, De Boer H, Doughty J, Myon E, Taieb C. Patients' understanding of and adjustment to epilepsy: interim findings from a European survey. Epilepsia. 1999; 40 Suppl 9: S26-S29.

77. Ford CA, Gibson P, Dreifuss FE. Psychossocial considerations in childhood epilepsy. In: Dreifuss FE, editor. Pediatric epileptology classification and management of seizures in childhood. Massachussets: John Wright and Sons; 1983: 277-95.

78. Brothers L. Friday's Footprint. How society shapes the human mind. Oxford, New York; 1997.

79. Moll J, Oliveira-Souza R, Bramati IE, Grafman J. Functional networks in emotional moral and nonmoral social judgments. Neuroimage. 2002; 16(3 Pt 1):696-703.

80. Damasio AR. Em busca de Espinosa: prazer e dor na ciência dos sentimentos. São Paulo: Editora Schwarcz, Companhia das Letras; 2004.
81. Moll J, Oliveira-Souza R, Eslinger PJ, Bramati IE, Mourao-Miranda J, Andreiuolo PA et al. The neural correlates of moral sensitivity: a functional magnetic resonance imaging investigation of basic and moral emotions. J Neurosci. 2002; 22 (7):2730-6.

82. Moll J, Oliveira-Souza R, Moll FT, Ignacio FA, Bramati IE, Caparelli-Daquer EM et al. The moral affiliations of disgust: a functional MRI study. Cogn Behav Neurol. 2005; 18(1):68-78.

83. Greene JD, Nystrom LE, Engell AD, Darley JM, Cohen JD. The neural bases of cognitive conflict and control in moral judgment. Neuron. 2004; 44(2):389-400.

84. Bartels A, Zeki S. Functional brain mapping during free viewing of natural scenes. Hum Brain Mapp. 2004; 21(2):75-85.

85. Ochsner KN, Ray RD, Cooper JC, Robertson ER, Chopra S, Gabrieli JD et al. For better or for worse: neural systems supporting the cognitive down- and up-regulation of negative emotion. Neuroimage. 2004; 23(2):483-99.

86. Logothetis N.K., Pfeuffer J. On the nature of the BOLD fMRI contrast mechanism. Magnetic Resonance Imaging. 2004; 22: 1517-31.

87. Westbrook C, Kaut C. Ressonância Magnética Prática. 2aㅡ ed. Rio de Janeiro: Guanabara Koogan; 1993.

88. Smith SM, Beckmann CF, Ramnani N, Woolrich MW, Bannister $\mathrm{PR}$, Jenkinson $\mathrm{M}$ et al. Variability in $\mathrm{fMRI}$ : a re-examination of inter-session differences. Hum Brain Mapp. 2005; 24(3):248-57.

89. Fernandes PT, Rio PA, Li LM. Functional magnetic resonance imaging study of stigma perception. Neuroimage. 2006; 31(1):S175. (Abstract)

90. Doughty J, Baker GA, Jacoby A, Lavaud V. Cross-cultural differences in levels of knowledge about epilepsy. Epilepsia. 2003; 44(1):115-23.

91. Fernandes PT, Salgado PC, Noronha AL, Mory SB, Rio PA, Li LM. Combate ao estigma na epilepsia pela conscientização através da mídia. Journal of Epilepsy and Clinical Neuropshysiology. 2004; 10(3):167-70.

92. Fernandes PT, Salgado PC, Noronha AL, Mory SB, Li LM. Formação de grupos como suporte psicológico e social na epilepsia. Journal of Epilepsy and Clinical Neuropshysiology. 2004; 10(3):171-4.

93. Risdale L. The effect of specially trained nurses in primary care: a review. Seizure. 2000; 9(1):43-6.

94. Kendall S, Thompson D, Couldridge L. The information needs of carers of adults diagnosed with epilepsy. Seizure. 2004; 13:499-508.

95. Kale R. Global Campaign Against Epilepsy:the treatment gap. Epilepsia. 2002; 43 Suppl 6:31-3.

96. Mattson RH. Medical management of epilepsy in adults. Neurology. 1998; 51(5 Suppl 4):S15-S20.

97. Ridsdale L, Robins D, Fitzgerald A, Jeffery S, McGee L. Epilepsy in general practice: patients' psychological symptoms and their perception of stigma. Br J Gen Pract. 1996; 46(407):365-6.

98. Fernandes PT, Cabral P, Araújo UF, Noronha ALA, Li LM. Kids' perception about epilepsy. Epilepsy Behav. 2005; 6(4):601-3.

\section{Endereço para correspondência:}

Paula T. Fernandes

Cx. Postal 6126,

CEP 13083-970, Campinas, SP, Brasil

Fone/Fax: (19)3521-7292

E-mail:paula@aspebrasil.org-www.aspebrasil.org 